\title{
Design para mudança de comportamento: Uma revisão sistemática nas conferências da Design Research Society (DRS)
}

Design for behavior change: A systematic review at Design Research Society (DRS) conferences.

ALMEIDA, Henrique Bittencourt; Mestrando; Universidade do Vale do Rio dos Sinos bittencurt.henrique@gmail.com

SCALETSKY, Celso Carnos; Professor Doutor; Universidade do Vale do Rio dos Sinos celsocs@unisinos.br

\section{Resumo}

O design para mudança de comportamento (DfBC) é uma abordagem que busca mudar o comportamento das pessoas, através de estratégias de design. O presente estudo procura identificar o que já foi publicado sobre o design para mudança de comportamento nas conferências da Design Research Society (DRS), com o intuito de fortalecer e contribuir com a estruturação teórica do campo. Como método de pesquisa, foi feita uma revisão sistemática em artigos publicados nas conferências de 2010 a 2016 do evento. A partir dessa revisão sistemática identificou-se algumas lacunas, onde futuras pesquisa sobre o design para mudança de comportamento, podem explorar a relação entre designers e os atores envolvidos no processo de projeto. Outra possibilidade que se revela, é a de desenvolver novos métodos projetuais que auxiliem designers a projetarem de maneira mais efetiva para a mudança de comportamento.

Palavras Chave: design para mudança de comportamento; revisão sistemática; mudança de comportamento e design research society.

\begin{abstract}
Design for behavior change ( $D f B C)$ is an approach that seeks to change people's behavior through design strategies. This study seeks to identify what has already been published about design for behavior change at Design Research Society (DRS) conferences, in order to strengthen and contribute to the theoretical structuring of the field. As a method of analysis, the systematic review was used in articles published in the conferences from 2010 to 2016. From this systematic review has identified some gaps, where future research on design for behavior change can observe, as the relationship between designers and the actors involved in the design process. Another possibility that has emerged throughout the review is that there are still many possibilities for researching and developing new design methods that will help designers to create methods more effectively for behavior change.
\end{abstract}

Keywords: design for behavior change; systematic review; behavior change and design research society. 


\section{Introdução}

Pesquisas e teorias sobre mudança de comportamento já existem há algumas décadas, porém, a cada ano surgem novas investigações que abrem espaço para outros debates. As ciências comportamentais abordam amplamente o estudo do comportamento humano, com base nas teorias da economia, psicologia e neurociência. (NIEDDERER et al., 2014). Porém, com o surgimento de tecnologias e produtos, novos estudos têm explorado o tema em colaboração com outras áreas do conhecimento, tais como o design. Dessa forma, abre-se a possibilidade para profissionais desenvolverem projetos que visam estimular a mudança de comportamento. Contudo, a complexidade em projetar novos artefatos que medeiam as relações humanas e seus comportamentos revela que o design necessita, entretanto, do apoio de outros campos do conhecimento e, também, de novos métodos de projetação. (FOGG, 2009).

A partir dos modelos e das bases teóricas da psicologia, economia e neurociência, pesquisadores como Fogg (2009), Wendel (2014) e Niedderer et al. (2014) perceberam que seria possível projetar artefatos com o objetivo de mudar o comportamento do usuário, por meio do design, e, a partir dessas perspectivas, começaram a formular suas teorias e seus métodos. Diante disso, surgiu o campo denominado de design para mudança de comportamento - referenciado a partir de agora pela sigla "DfBC", originada do termo inglês "Design for Behavior Change". De forma sintética, estes definem o DfBC como uma abordagem que objetiva influenciar 0 comportamento dos usuários por meio de estratégias de design que buscam incentivar as práticas humanas desejáveis. (BOKS, 2011; CASH; GRAM; BOYSEN, 2017; CLUNE, 2010; LILLEY; LOFTHOUSE, 2009; LOCKTON et al. 2013; NIEDDERER, 2013; NIEDDERER et al. 2014; WENDEL, 2014).

De acordo com Niedderer et al. (2014), foi na década de 1980 que surgiu um dos primeiros estudos sobre design e mudança de comportamento, quando Don Norman introduz o termo design comportamental em uma obra denominada "The Psychology of Every Things", que explora as particularidades cognitivas dos usuários que precisam ser consideradas em projetos de produto, fornecendo princípios orientadores para o design projetar artefatos de uso intuitivo. Apesar de ser uma obra influente na área, que contribui de forma significativa para a propagação dos conceitos da psicologia no design, seu mentor não discutiu de forma aprofundada as estratégias que estimulam a mudança comportamental. Pouco tempo depois, mais especificamente a partir do final dos anos de 1990, surgiram os primeiros estudos sobre o DfBC. (LOCKTON; HARRISON; STANTON, 2010). Em um editorial para a revista "Designing for Consumers", Stanton e Baber (1998) sinalizaram que, designers, ao projetarem produtos, estão também projetando a atividade dos usuários que os utilizam e acabam, consequentemente, moldando o comportamento desses usuários.

Apesar da clara influência do design sobre o comportamento humano, o DfBC possui poucos estudos e só foi reconhecido formalmente como um campo do conhecimento na última década. (NIEDDERER et al., 2014). Diante disso, o objetivo desse estudo é identificar e analisar o que já foi publicado nas conferências da Design Research Society (DRS) sobre o DfBC, com o intuito de fortalecer e contribuir com a estruturação teórica desse campo. Essa conferência foi criada em 1966, e tem como objetivo promover o desenvolvimento da pesquisa de design.

Para alcançar esse objetivo, foi realizada uma revisão sistemática, onde observou-se os seguintes itens: (i) quem são os autores mais citados na área, (ii) quais são as ferramentas, modelos e métodos projetuais criados ou abordados nos trabalhos analisados e, por fim (iii) que 
tipo de lacunas que a área apresenta para futuras pesquisas. Após a apresentação do tema e dos objetivos, será apresentado o método utilizado no presente artigo. Posteriormente, os resultados são apresentados e discutidos e, por fim, as considerações finais são desenvolvidas.

\section{Método}

Para responder os objetivos propostos, optou-se por utilizar a técnica de revisão sistemática. De acordo com Khan et al. (2003) uma análise pode ser considerada sistemática se for construída a partir de uma metodologia explícita e organizada, que se propõe a responder um problema bem definido por meio da identificação de pesquisas relevantes sobre ela, para depois avaliar a qualidade das pesquisas e sintetizar as evidências encontradas.

Para Khan et al. (2003) uma análise sistemática rigorosa deve ser desenvolvida em cinco passos: (1) enquadramento da questão a ser investigada, neste caso, o que já foi publicado sobre DfBC na conferência da Design Research Society (DRS); (2) identificação de trabalhos relevantes, aqui desenvolvido com a busca por artigos na conferência da Design Rearch Society, nos anos de 2010, 2012, 2014 e 2016; (3) avaliação da qualidade dos estudos, indicada pela escolha dos periódicos revisados por pares e encontrados a partir da definição de palavras chaves, e posterior análise detalhada dos textos; (4) resumo das evidências e (5) interpretação dos achados, desenvolvido nos itens da Discussão e das Considerações Finais.

Para esta pesquisa, as etapas de seleção e análise foram: (a) listagem das edições do evento, (b) obtenção dos proceedings do evento e seleção dos artigos classificados na temática do DfBC, (c) leitura de resumos dos artigos encontrados, (d) triagem dos artigos que realmente pertenciam ao tema, (e) leitura dos artigos considerados e resumo dos seus dados e (f) análise de dados obtidos. Todos procedimentos de coleta, triagem, seleção e leitura dos artigos foram listados em uma planilha de dados, na qual foram registrados ano, volume do proceeding, página do artigo, autor, instituição de ensino de origem, título do trabalho, palavras-chave. Em relação a seleção dos artigos, por se tratar de uma conferência onde todos os artigos estão em inglês, procurou-se nos títulos dos artigos de todas as edições revisadas e nas palavras chaves, os termos design for behavior change ou behavior change. A tabela 1 reúne os resultados das buscas.

Tabela 1 - Artigos submetidos por edição da conferência Design Research Society

\begin{tabular}{llll}
\hline Edição / Ano & Total de artigos & Artigos relacionados ao DfBC & Artigos sobre DfBC considerados \\
\hline 2010 & 150 & 5 & 0 \\
2012 & 154 & 6 & 0 \\
2014 & 132 & 8 & 3 \\
2016 & 240 & 15 & 7 \\
\hline TOTAL & 676 & 34 & 10 \\
\hline
\end{tabular}

Fonte: elaborado pelo autor, com base na pesquisa realizada

Ao todo, somaram-se 676 artigos nas edições de 2010 a 2016 nas conferências da design research society (DRS). Escolheu-se esse recorte temporal, pois era preciso identificar os estudos 
atuais sobre a temática. Além disso, também não foi possível acessar os proceedings das edições anteriores do evento. Após a análise nos títulos e palavras-chaves de todos os artigos, foram descartados os artigos que não possuíam nenhuma relação com o tema, restando 34 artigos que tratavam de temas relacionados à mudança de comportamento. Dessa forma, ocorreu a leitura dos resumos, no qual foram considerados 10 artigos que tratavam diretamente sobre o DfBC. Após leitura completa dos estudos, foi feita a leitura e discussão sobre cada um dos artigos e apresentada na seção seguinte.

\section{Resultados}

Nesta seção são descritos e apresentados os resultados alcançados a partir dos critérios apresentados na seção anterior. Optou-se por apreciar os textos e resgatá-los um a um em ordem crescente. Para um melhor entendimento do leitor, pode-se conferir a quadro 1 , com o ano, 0 título do artigo e seus autores.

Quadro 1 - Resumo dos artigos selecionados

\begin{tabular}{|c|c|c|}
\hline Ano & Título do artigo & Autores \\
\hline 2014 & User Diversity in Design for Behavior Change & Coskun, A.; Erburg, C \\
\hline 2014 & $\begin{array}{l}\text { Communication design as an agent in creating gender equality in } \\
\text { India }\end{array}$ & John, N. \\
\hline 2014 & $\begin{array}{l}\text { Social Implication Design (SID): A design method to exploit the } \\
\text { unique value of the artefact to counteract social problems }\end{array}$ & Tromp, N.; Hekkert, P. \\
\hline 2016 & $\begin{array}{l}\text { An alternative approach to influencing behaviour: Adapting } \\
\text { Darnton's Nine Principles framework for scaling up individual } \\
\text { upcycling }\end{array}$ & Sung, K.; Cooper, T.; Kettley, S. \\
\hline 2016 & $\begin{array}{l}\text { The potential of Design for Behaviour Change to foster the } \\
\text { transition to a circular economy }\end{array}$ & Piscicelli, L.; Ludden, G. \\
\hline 2016 & $\begin{array}{l}\text { Developing a theory-driven method to design for behaviour } \\
\text { change: two case studies }\end{array}$ & $\begin{array}{l}\text { Essen, V.; Hermsen, S.; Renes, } \\
\text { R. }\end{array}$ \\
\hline 2016 & $\begin{array}{l}\text { What a designer can change: a proposal for a categorisation of } \\
\text { artefact-related aspects }\end{array}$ & $\begin{array}{l}\text { Selvefors, A.; Stromberg, H.; } \\
\text { Renstrom, S. }\end{array}$ \\
\hline 2016 & $\begin{array}{l}\text { Exploring and communicating user diversity for behavioural } \\
\text { change }\end{array}$ & Coskun, A.; Erburg, C. \\
\hline 2016 & $\begin{array}{l}\text { How I learned to appreciate our tame social scientist: experiences } \\
\text { in integrating design research and the behavioural sciences }\end{array}$ & $\begin{array}{l}\text { Hermsen, S., Van Der Lugt., } \\
\text { Mulder, S., Renes, R. J. }\end{array}$ \\
\hline
\end{tabular}


Coskun e Erbug (2014) argumentam que um dos aspectos centrais DfBC, é definir a estratégia de projeto correta para os comportamentos e usuários certos. O estudo estabelece uma base inicial como fonte de informação, propondo uma estrutura para a escolha de estratégias de design. Para os autores a diversidade de usuários pode ser abordada agrupando-os com características semelhantes em diferentes tipos de usuários. A partir dessa constatação, são elaborados um quadro e uma metodologia para definir quais são esses tipos de usuários com base em variáveis psicológicas, que incluem a atitude ambiental global, atitude em relação ao comportamento, normas subjetivas, controle comportamental percebido, intenção e por fim, traços de personalidade. No quadro, os designers devem definir um objetivo de design bem estruturado, conhecer seus usuários-alvo e estar atentos às estratégias de intervenção de projeto disponíveis para eles. Como resultado do artigo, são propostos quatro tipos de usuários principais: usuários irresponsáveis, usuários indecisos, usuários preocupados e usuários entusiasmados. Nesse sentido, as estratégias de intervenção de design são combinadas com esses tipos de usuários e o artigo termina com uma breve discussão sobre as implicações da estrutura e da metodologia para o DfBC.

John (2014) a partir dos conceitos do DfBC, investigou se o design na comunicação é uma ferramenta efetiva na mudança de comportamentos culturais e percepções de gênero na Índia. De acordo com o autor, estudos anteriores sobre campanhas de violência de gênero sugerem que a utilização de homens e os estereótipos tradicionais de gênero são eficazes na criação de mudanças de comportamento na Índia. Contudo, John (2014) percebeu que ainda existe uma lacuna nos papéis culturais específicos e nos comportamentos enraizados do país. Portanto, o estudo tem como objetivo compreender o necessário das tradições e comportamentos culturais que devem preceder qualquer atividade de design dentro deste cenário. $\mathrm{O}$ artigo foi dividido em duas etapas de investigação. Na primeira parte, os participantes indianos masculinos foram entrevistados sobre a igualdade de gênero na Índia. Na segunda etapa, os participantes completaram kits de auto documentação, que se concentraram nas percepções de gênero. Após a análise das entrevistas, percebeu-se que a pesquisa obteve Insights que indicaram que o termo igualdade de gênero foi mal interpretado pelos entrevistados, pois grande parte dos entrevistados acreditavam que a Índia era uma sociedade muito igual para homens e mulheres. Nesse sentido, o artigo dá subsídios aos designers para projetarem uma campanha de comunicação culturalmente relevante, podendo enfrentar a violência de gênero de uma perspectiva privilegiada e promover a mudança de comportamento através do design dentro do contexto indiano.

Tromp e Hekkert (2014) elaboraram um método chamado de Social Implication Design (SID). O objetivo desse método consiste em apoiar o designer na reflexão acerca de um problema social, para que, então, ele desenvolva uma solução de design que impacte de forma positiva e promova a mudança de comportamento em grupos da sociedade. Nesse sentido, o foco do método está nas consequências sociais que o projeto irá gerar, já que as preocupações individuais dos usuários, muitas vezes, estão em conflito com os propósitos coletivos da sociedade. (TROMP; HEKKERT, 2014). Diante dessa constatação, o modelo coloca o designer como o principal agente 
mediador dessa problemática, propondo etapas no processo de projeto que contribuam para gerar soluções positivas para o coletivo. O método proposto pelos autores possui cinco etapas: (a) identificação do fenômeno social a ser revertido ou minimizado; (b) definição do efeito social desejado; (c) definição do comportamento pretendido; (d) criação de estratégia que motive os usuários por intermédio do design; e (e) materialização da ideia em propostas de design. Nessa última etapa, é possível ao designer, caso necessário, agregar outros métodos ao processo projetual. Contudo, vale ressaltar que o resultado do projeto não necessariamente consiste em um artefato. Ao final do artigo, os autores sugerem que os designers realizem uma avaliação dos resultados obtidos, com o intuito de medir o potencial de influência da mudança de comportamento e de obter a implicação social desejada. (TROMP; HEKKERT, 2014).

Piscinelli e Ludden (2016) discutem como o DfBC com foco no design para comportamento sustentável, poderia contribuir para abordar e promover a transição para uma economia circular. A economia circular pode ser definida como "uma economia industrial restaurativa em suas intenções, que permite fluxos eficazes de materiais, energia, trabalho e informação para que o capital social possa ser reconstruído". (MACARTHUR, 2013, p. 26). Para Piscinelli e Ludden (2016), a pesquisa sobre a economia circular no contexto do design, tem sido amplamente limitada à identificação de estratégias de design para modelos, produtos e serviços de negócios circulares. De acordo com os mesmos autores, aplicar os conceitos do DfBC na economia circular poderia garantir uma melhor compreensão do comportamento do consumidor e as condições de habilitação para alcançar mais aceitação em relação a modelos de negócios circulares. Em particular, utilizar os conceitos do DfBC poderia oferecer informações valiosas sobre como estimular a demanda por produtos remanufaturados, produtos de segunda mão e como incentivar atividades de reparação e manutenção que poderiam ajudar a prolongar a vida útil do produto.

Sung, Cooper e Kettley (2016) revisaram criticamente a estrutura de nove princípios de Darnton como uma estrutura abrangente que aborda a necessidade de design e intervenção política. O framework dos nove princípios de Darnton foi projetado para integrar modelos de comportamento, com compreensão teórica de abordagens efetivas de mudança. A estrutura fornece um ponto de partida para selecionar modelos apropriados e desenvolver intervenções de mudança de comportamento. De acordo com Sung, Cooper e Kettley (2016) o framework sugere os nove principios como: (1) identificar os grupos de público (ou atores) e o comportamento do usuário; (2) identificar modelos comportamentais relevantes e elaborar uma lista restrita de fatores influenciadores; (3) selecionar os principais fatores de influência para projetar objetivos de intervenção; (4) identificar técnicas de intervenção efetivas; (5) envolver o usuário na intervenção; (6) desenvolver um protótipo dessa intervenção; (7) observar a intervenção e monitorar continuamente; (8) avaliar os impactos e processos; e por fim, (9) aprender com a avaliação. Os autores concluem acreditando que as intervenções geradas a partir desta abordagem podem ser eficazes em termos de impacto na mudança de comportamento, porque é baseada em modelos de comportamento testados e evidências de pesquisa científica. Dessa forma poderia fornecer aos designers uma certa garantia de que as intervenções, quando testadas ou implementadas, criariam um impacto positivo em que pretende mudar seu comportamento.

Essen, Hermsen e Renes (2016) analisaram dois estudos de caso de design, onde buscaram responder a seguinte pergunta: como podem ser incorporadas as teorias e evidências das ciências comportamentais em um método de design de tal forma que isso não prejudique a criatividade? De acordo com os autores, o DfBC tem muito a ganhar com a integração de insights das ciências 
comportamentais no processo de design. No entanto, essa integração precisa ser feita sem prejudicar o processo criativo. No estudo elaborado, foram realizados dois estudos de caso, onde foi elaborado um método chamado de Persuasive by Design-model (PbD) com base no modelo do Double Diamond. Os estudos de caso demonstraram que o método realmente aumenta a integração da teoria nos projetos, mas apenas se o modelo de processo Double Diamond for complementado com uma fase de avaliação. Para dar suporte à utilização do modelo, os autores desenvolveram uma ferramenta denominada Behavioural Lenses toolkit. Essa ferramenta é apresentada em um folheto, onde cada uma das lentes é acompanhada por um subconjunto de questões relacionadas à mudança de comportamento e por referências relevantes na literatura, fornecendo, também, estratégias persuasivas. Essa ferramenta pode ser usada tanto para informar a pesquisa dos usuários quanto para avaliar os conceitos de projeto.

Selvefors, Strömberg e Renström (2016) propõem uma categorização de aspectos relacionados com artefatos adaptados ao design para comportamento sustentável. Segundo os autores, o design para comportamento sustentável, surgiu como um importante campo de pesquisa e prática, abordando desafios de sustentabilidade relacionados ao uso de produtos, serviços e sistemas pelas pessoas. Nesse tipo de abordagem, o design é usado para encorajar e possibilitar novas formas de fazer e interagir com o mundo que, por sua vez, contribuem para a sociedade. A categorização dos artefatos, pode servir como ponto de partida para discutir quais aspectos são relevantes para considerar a partir de uma perspectiva de design para o comportamento sustentável. A categorização classifica esses aspectos em diferentes camadas: da camada abrangente da atividade habilitada, através de tipos de artefato, funções operacionais, funções interativas e, finalmente, funções comunicativas. O artigo também discute os benefícios de redesenhar os artefatos que as pessoas usam em suas atividades diárias ao invés de projetar artefatos que estimulam novos tipos de atividades de conservação.

Coskun e Erbug (2016) enfatizam a importância da diversidade de usuários para mudanças comportamentais. No estudo, é proposto um método e mapas de orientação do usuário, para explorar a sua diversidade e, comunicar essa diversidade aos designers. Para avaliar sua aplicabilidade em diferentes contextos de mudanças comportamentais, foram realizados dois estudos de caso. $O$ primeiro estudo explorou a diversidade nas orientações dos usuários para a adaptação de um estilo de condução ecológico. O segundo estudo explorou a diversidade de usuários para adaptar o uso de um sistema urinário inteligente que permite que os pacientes aprendam o risco de ter Hiperplasia Prostática Benigna (BPH) sem visitar um hospital. Após análise, entendeu-se que o método proposto possui flexibilidade suficiente para ser aplicado em diferentes contextos de mudanças comportamentais. Para o futuro, os autores pretendem ampliar os domínios de aplicação dos mapas de orientação do usuário, comparar duas abordagens diferentes para construí-lo e avaliar sua utilidade e qualidade para os designers.

Hermsen et al. (2016) abordam à falta de integração da pesquisa em design com os cientistas comportamentais. Para os autores, ainda não houve muitas tentativas de investigar processos projetuais interdisciplinares no DfBC que envolvam designers e cientistas comportamentais. A partir dessa questão, os autores elaboram um workshop com um grupo multidisciplinar de cientistas comportamentais e pesquisadores de design, para identificar possíveis sinergias, bem como barreiras e limiares que impediram uma cooperação perfeita. Como resultado percebeu-se que os pesquisadores do projeto se beneficiaram das possibilidades de ancoragem do processo de design na teoria e evidenciaram a abordagem científica 
comportamental oferecida. Já os cientistas comportamentais se beneficiaram do aumento da validade ecológica da perspectiva conceituada. Se realizado com cuidado e consideração, uma abordagem conjunta combinando métodos conceituais com ciência comportamental pode, portanto, aumentar significativamente a eficácia do trabalho de todas as partes envolvidas. No entanto, ocorreram diferenças que podem dificultar a sinergia. Diferenças no que constitui a evidência e como a verdade é encontrada podem facilmente levar a diferenças intransponíveis na abordagem, dificultando a avaliação e a contribuição entre os participantes do workshop. Para Hermsen et al. (2016) os benefícios mais surpreendentes dessa cooperação não foram para os pesquisadores de design ou para os cientistas comportamentais envolvidos, mas para seus clientes.

Ludden e Ruijter (2016) investigam como mudar os hábitos alimentares (lanches) das crianças e seus pais. Neste artigo, foi aplicado o modelo elaborado por Ludden e Hekkert (2014) chamado de modelo de design para o comportamento saudável. O modelo em questão revela diferentes níveis de envolvimento e motivação do indivíduo em mudar seus hábitos. Segundo esse modelo, o usuário, ao iniciar esse processo de transformação, geralmente está com pouco interesse em mudar seu comportamento, apresentando, como consequência, um nível de motivação baixo. Contudo, ao longo dos estágios da mudança comportamental, o indivíduo vai aumentando seu engajamento, até que, por fim, acabe incorporando o novo comportamento à sua rotina. Este artigo tem dois objetivos principais. Em primeiro lugar, servir como uma demonstração sobre como o design para o comportamento saudável pode ser usado como guia de design. Em segundo lugar, avaliar até que ponto os produtos e serviços que foram projetados são de fato particularmente adequados para pessoas em estágios específicos de mudança. A partir disso foram elaborados dois diferentes projetos, que buscavam contemplar cada um dos estágios de mudança comportamental. Após isso, esses projetos foram descritos e colocado on-line para uma pesquisa com os pais de crianças de 2 a 7 anos. A partir da obtenção dos dados, foi possível descobrir que os participantes esperavam que alguns desses exemplos tivessem influência sobre a consciência de seus filhos sobre a importância da alimentação saudável. Além disso, os pais esperavam que alguns desses exemplos tivessem um efeito sobre seu comportamento em relação aos lanches. Outra conclusão importante é que, embora os pais avaliem lanches saudáveis e aprendam sobre o comportamento alimentar saudável, muitas vezes não estão dispostos ou são capazes de investir uma quantidade considerável de tempo nisso.

\section{Discussão}

A discussão do trabalho, se organiza a partir dos objetivos propostos no método: (i) quem são os autores mais citados na área, (ii) quais são as ferramentas, modelos e métodos projetuais criados ou abordados nos trabalhos analisados e, por fim (iii) que tipo de lacunas que a área apresenta para futuras pesquisas.

Antes de falar sobre os autores e referências mais citados, é preciso ressaltar que o DfBC é um campo que ainda está se construindo. Portanto, existem poucos autores que se dedicam a pesquisar e escrever frequentemente sobre o tema, fazendo com que a área ainda não tenha seus principais autores definidos. Contudo, foi possível encontrar citações a teoria de autores como Fogg (2003) e Fogg (2009) que é considerado um dos precursores sobre os estudos da tecnologia persuasiva, campo que pertence ao DfBC. Outros autores em que há citações sobre suas teorias são Wendel (2014), Niedderer (2014), Ludden e Hekkert (2014), Lockton, Harrison e Stanton (2010) e Lilley (2009). 
Sobre as ferramentas, modelos e métodos projetuais que são criados ou utilizados nos estudos, pode-se perceber nessa análise sistemática que, por ser um campo ainda recente, os autores estão criando e testando muitas ferramentas, modelos e métodos projetuais que possam auxiliar designers ao longo do processo de projeto. Percebe-se que os autores que escrevem sobre o DfBC ainda estão explorando o campo da psicologia para tentar assimilar as teorias e aplicá-las ao contexto do design. Nesse artigo, um dos modelos encontrados foca exclusivamente na importância da diversidade de usuários para mudanças comportamentais e elabora estratégias que possam auxiliar os designers a identificá-los em diferentes grupos. (COSKUN; ERBURG, 2014; COSKUN; ERBURG, 2016).

Foi analisado também um estudo que testa um modelo embasado nas teorias da psicologia que tem como objetivo auxiliar projetos que visem o comportamento saudável e bem-estar das pessoas. (LUDDEN; RUIJTER, 2016). Outro estudo analisado, apresenta um método que tem por objetivo apoiar o designer na reflexão acerca de um problema social. (LUDDEN; HEKKERT, 2016). De acordo com Niedderer (2014), estudos que tratam sobre esse tema no DfBC, são raros. Niedderer (2014) afirma que o design para o comportamento sustentável é a área do DfBC que possui mais estudos e também um maior reconhecimento por parte da academia. Por fim, foi apresentado um modelo chamado Persuasive by Design model (PbD), com o objetivo de criar intervenções mais efetivas de mudança de comportamento por meio do design para uma vida mais sustentável e saudável. No entanto, constatou-se que o uso efetivo desse modelo é dificultado pela sua complexidade. (HERMSEN; RENES, 2016).

No presente estudo, identificou-se também uma lacuna que possibilita futuras pesquisas nesse campo. Constatou-se que os modelos e métodos até então estabelecidos no DfBC, não incluíam todos os atores (usuário, designer e profissionais de outras áreas do conhecimento) no processo de projeto. Os projetos de design para mudança de comportamento estão utilizando as teorias das ciências comportamentais apenas em uma fase preliminar de ideação como fonte de inspiração, o que, por consequência, faz com que nas etapas seguintes do projeto, essas teorias fiquem perdidas em meio às ideias, aos conceitos e aos outros processos de projeto (HERMSEN et al., 2016). Outra problemática levantada, concerne à falta de integração da pesquisa em design com os cientistas comportamentais. Para os autores, ainda não houve muitas tentativas de investigar processos projetuais interdisciplinares no DfBC que envolvam designers e cientistas comportamentais (HERMSEN et al., 2016). Diante disso, futuras pesquisa sobre o DfBC, podem observar a relação entre designers e atores envolvidos no projeto de design para mudança de comportamento. Além disso, também seria possível elaborar estratégias que possibilitem essa integração em todas etapas projetuais.

\section{Considerações Finais}

O objetivo geral dessa análise sistemática, era identificar publicações sobre o DfBC, nas conferências da Design Research Society (DRS) com intuído de encontrar possíveis lacunas para futuras pesquisa. Nesse sentido, a revisão sistemática se mostrou relevante, identificando artigos relacionados com a temática estabelecida, apresentando dez publicações que possuíam uma ligação direta com DfBC. Diante disso, foi possível perceber que até setes anos atrás, as publicações sobre esse campo no DRS, eram nulas. Porém, a partir de 2014, esse número foi aumentando até que em dois mil e dezesseis, o evento teve uma seção exclusiva com artigos sobre essa temática, mostrando que a tendência nas próximas conferências é de cada vez mais surgirem estudos sobre o DfBC. 
Com relação aos objetivos da análise sistemática, acredita-se que os achados podem possibilitar novos conhecimentos acerca da temática. Identificou-se que os principais autores do DfBC foram citados constantemente nos artigos, o que mostra que o campo vem se construindo com bases sólidas. A respeito da criação ou utilização de ferramentas, modelos ou métodos projetuais, percebe-se que grande parte dos artigos analisados tinham como foco testar, propor ou validar esse tipo estudo. Isso demonstra que em futuras pesquisa pode-se pesquisar e desenvolver novos métodos projetuais que auxiliem designers a projetarem de maneira mais efetiva para a mudança de comportamento.

\section{Referências}

BOKS, C. Design for Sustainable Behaviour Research Challenges. Proceedings of EcoDesign 2011 International Symposium, p. 328-333, 2011.

CASH, P. J.; GRAM, C. H.; BOYSEN, C. D. Behavioural design: A process for integrating behaviour change and design. Design Studies, v.48, p. 96-128, 2017.

CLUNE, S. Design for Behavioral Change. The Journal of Design strategies, v.4, n. 1, p. 68-75, 2010.

COSKUN, A.; ERBURG, C. Exploring and communicating user diversity for behavioural change. In: PROCEEDINGS OF DRS, 2016, Brighton, UK. v. 4, p. 1357-1374, 2016.

COSKUN, A.; ERBURG, C. User diversity in design for behavior change. In: PROCEEDINGS OF DRS, 2014, Umea, Sweden. v. 1, p. 546-559, 2014.

MACARTHUR, Foundation. Towards the Circular Economy. The Ellen MacArthur Foundation Report, p. 26, 2013.

ESSEN, V.; HERMSEN, S.; RENES, R. Developing a theory-driven method to design for behaviour change: two case studies. In: PROCEEDINGS OF DRS, 2016, Brighton, UK. v. 4, p. 1323-1337, 2016.

FLUSSER, V. O mundo codificado. Por uma Filosofia do design e da comunicação (1ạ ed.) São

Paulo: Cosac Naify, 2007.

FOGG, B. J. A Behavior Model for Persuasive Design. In: Proceedings of the 4th international conference on persuasive technology, 2009, Claremont (CA). Anais..., Claremont (CA), ACM, 2009. Art. 40.

HERMSEN, S., VAN DER LUGT., MULDER, S., RENES, R. J. How I learned to appreciate our tame social scientist: experiences in integrating design research and the behavioural sciences. in: $P$. Lloyd \& E. Bohemia, eds., Proceedings of DRS2016: Design + Research + Society - Future-Focused Thinking, v. 4, p. 1375-1389, 2016.

JELSMA, J. Designing 'Moralized' Products: Theory and Practice. In: VERBEEK, P. P.; SLOB, A. (Eds.). User Behavior and Technology Development: Shaping Sustainable Relations Between Consumers and Technologies. Dordrecht, the Netherlands: Springer, p. 221-231, 2006.

JOHN, N. Communication design as an agent in creating gender equality in India. In: PROCEEDINGS OF DRS, 2014, Umea, Sweden. v. 1, p. 530-545, 2014.

KRIPPENDORFF, K. The semantic turn. A new foundation for design. Boca-Raton: Taylor \& Francis, 2006.

LILLEY, D.; LOFTHOUSE, V. Teaching Ethics For Design For Sustainable Behaviour: A pilot study. 
Design and Technology Education: An International Journal. v.15, n.2: 55-68, 2009.

LOCKTON, D., HARRISON, D., STANTON, N. Design with Intent: 101 Patterns for Influencing Behaviour Through Design. Tradução de Luiz Oliveira. v.1.0, Windsor: Equifine, 2010a.

LOCKTON, D.; HARRISON, D.; STANTON, N. Exploring Design Patterns for Sustainable Behaviour. The Design Journal, London, v.16, n. 4, p. 431-459, 2013.

LUDDEN, G.; HEKKERT, P. Design for healthy behavior design interventions and stages of change. Proceedings of the Colors of Care: The 9th International Conference on Design \& Emotion. Ediciones Uniandes, Bogotá, p. 482-488, 2014.

LUDDEN, G.; RUIJTER, L. Supporting healthy behaviour: A stages of change perspective on changing snacking habits of children. In: PROCEEDINGS OF DRS, 2016, Brighton, UK. v. 4, p. 14741486, 2016.

MORANDI, M.; CAMARGO, L. (2014). Revisão Sistemática da Literatura. Design Science Research: método de pesquisa para avanço da ciência e tecnologia. RS, 10 edição, Editora Bookman, pag. 141-172.

NIEDDERER, K. et al. Creating Sustainable Innovation through Design for Behaviour Change: Full Project Report. Leicestershire, UK: Project Partners \& AHRC, 2014.

NIEDDERER, K. Mindful Design as a Driver for Social Behaviour Change. Consilence and Innovation in Design - Proceedings and Program, 2013.

NIEDDERER, K., MACKRILL, J., CLUNE, S., LOCKTON, D., LUDDEN, G., MORRIS, A., CAIN, R., GARDINER, E., GUTTERIDGE, R., EVANS, M., HEKKERT, P. Creating Sustainable Innovation through Design for Behaviour Change: Full Report. University of Wolverhampton, Project Partners \& AHRC, 2014.

PISCICELLI, L.; LUDDEN, G. The potential of Design for Behaviour Change to foster the transition to a circular economy. In: PROCEEDINGS OF DRS, 2016, Brighton, UK. v. 4, p. 1306-1321, 2016.

SELVEFORS, A.; STROMBERG, H.; RENSTROM, S. What a designer can change: a proposal for a categorisation of artefact-related aspects. In: PROCEEDINGS OF DRS, 2016, Brighton, UK. v. 4, p. 1340-1355, 2016.

STANTON, N. A.; BABER, C. “Designing for consumers: editorial.” Applied Ergonomics, v.29, no. 1, p. 1-3, 1998.

SUNG, K.; COOPER, T.; KETTLEY, S. An alternative approach to influencing behaviour: Adapting Darnton's Nine Principles framework for scaling up individual upcycling. In: PROCEEDINGS OF DRS, 2016, Brighton, UK. v. 4, p. 1277-1290, 2016.

TRANFIELD, D; DENYER, D; SMART, P. Towards a Methodology for Developing Evidence-Informed Management Knowledge by Means of Systematic Review. British Journal of Management, v. 14, p.207-222, 200. KHAN et al. Five steps to conducting a systematic review. Journal of the royal society of medicine, v. 96, p.118-121, 2003.

TROMP, N.; HEKKERT, P. Social Implication Design (SID) - A Design Method to Exploit the Unique Value of the Artifact to Counteract Social Problems. In: PROCEEDINGS OF DRS, 2014, Umea, Sweden. v. 1, p. 584-598, 2014. 
WENDEL, S. Designing for Behavior Change. O’Reilly Media, Inc., 2014. 